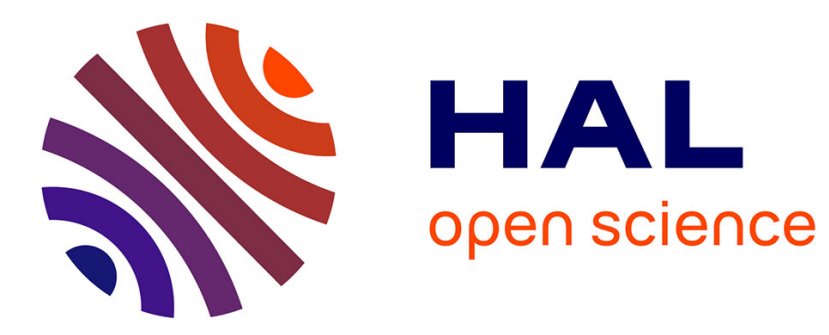

\title{
Long-time behaviour of particles diffusing in strongly disordered medium, revisited
}

\author{
Y. Shapir
}

\section{To cite this version:}

Y. Shapir. Long-time behaviour of particles diffusing in strongly disordered medium, revisited. Journal de Physique Lettres, 1984, 45 (18), pp.895-899. 10.1051/jphyslet:019840045018089500 . jpa00232428

\section{HAL Id: jpa-00232428 https://hal.science/jpa-00232428}

Submitted on 1 Jan 1984

HAL is a multi-disciplinary open access archive for the deposit and dissemination of scientific research documents, whether they are published or not. The documents may come from teaching and research institutions in France or abroad, or from public or private research centers.
L'archive ouverte pluridisciplinaire HAL, est destinée au dépôt et à la diffusion de documents scientifiques de niveau recherche, publiés ou non, émanant des établissements d'enseignement et de recherche français ou étrangers, des laboratoires publics ou privés. 
Classification

Physics Abstracts

$05.40-05.60-66.10$

\title{
Long-time behaviour of particles diffusing in strongly disordered medium, revisited
}

\author{
Y. Shapir $(*)$ \\ Department of Physics and Materials Research Laboratory, \\ University of Illinois at Urbana-Champaign, Urbana, Illinois 61801, U.S.A.
}

(Reçu le 12 octobre 1983, révisé le 19 juillet 1984, accepté le 24 juillet 1984)

\begin{abstract}
Résumé. - On réexamine l'argument heuristique de Lifshitz pour le comportement à longue durée de la diffusion classique dans le régime fortement désordonné. La déviation de la densité à l'origine suit un comportement en $\exp \left\{-c t^{\psi}\right\}$ avec $\psi=\tilde{d} /(2+\tilde{d})$, où $\tilde{d}$ est la dimension spectrale de l'amas. L'amas typique donne $\psi \approx 2 / 5$, mais on montre que le comportement asymptotique est dominé par des amas plus ramifiés avec $\tilde{d}=1$. Ceci produit un comportement typiquement unidimensionnel, $\psi=1 / 3$, en toute dimension.
\end{abstract}

\begin{abstract}
We reexamine the heuristic Lifshitz argument for the long-time behaviour of classical diffusion in the strongly disordered regime. The deviation of the density at the origin behaves as $\exp \left\{-c t^{\psi}\right\}$ with $\psi=\tilde{d} /(2+\tilde{d})$, where $\tilde{d}$ is the spectral dimension of the cluster. Typical clusters yield $\psi \approx 2 / 5$. We argue, however, that the long-time behaviour is dominated by more ramified clusters with $\tilde{d}=1$, leading to a one-dimensional behaviour, $\psi=1 / 3$, in any dimension.
\end{abstract}

Very recently there has been renewed interest in the classical diffusion on random percolating clusters, the so called " ant in the labyrinth " problem as formulated by de Gennes [1-3]. The new works focus on the anomalous behaviour on scales smaller than the correlation length $\xi$, where the percolating cluster has a Hausdorff (fractal) dimension $\bar{d}=d-\beta / v$ [4]. Gefen et al. [5] pointed out the non-trivial rescaling of the diffusion constant in this regime, related by scaling and Einstein relations to the conductivity exponent at the threshold. This yields a relation of the form :

$$
D_{0} t=L^{2+\theta}
$$

for the time $t$ it takes for the particle to diffuse at linear distance $L<\xi$. Recent numerical computations confirm this relation [6,7]. Alexander and Orbach [8] considered the density of classical and quantum modes on these clusters and defined the spectral dimension :

$$
\tilde{d}=2 \bar{d} /(2+\theta) \text {. }
$$

$\left(^{*}\right)$ Present address : Physics Department, Brookhaven National Laboratory, Upton, New York 11973, U.S.A. 
They outlined the numerical evidence of $\tilde{d} \approx 4 / 3$ at $p_{\mathrm{c}}$, in all dimensions (with exact equality for $d>6$ ). Rammal and Toulouse [9] have further analysed the relations between the random walk (RW) and the density of excitations on random and self-similar geometries.

The subject of the present communication concerns the long-time behaviour of the averaged density at the origin, $P_{0}(t)$, below the percolation threshold.

At $t=\infty$ the ultimate distribution, $P_{0}(\infty)$, is uniform and given by $\langle s\rangle^{-1}$, where $\langle s\rangle$ is the average over all clusters of their number of sites [4]. The asymptotic deviation from this value has been calculated for the one-dimensional case [10] :

$$
\Delta P_{0}(t)=P_{0}(t)-P_{0}(\infty) \sim \exp \left\{-\lambda t^{1 / 3}\right\}
$$

A first attempt to generalize this result to higher dimensions was made by Tanaka [11]. He finds :

$$
\Delta P_{0}(t) \sim \exp \left\{-c_{\mathrm{d}} t^{d /(2+d)}\right\} .
$$

This equation is derived from a heuristic argument à la Lifshitz [12] (see below) and from calculations based on the effective replicated Hamiltonian. However, in both derivations the crucial assumption is made that the compact clusters are making the most important contribution in the asymptotically long-time limit. The reasoning follows the one made to derive the density of states at the tail of the spectrum of a quantum particle in a random potential. For this case Lifshitz [12] first argued that the lowest energy states are dominated by contributions from the largest compact regions free from impurities; he then found :

$$
\rho(E)=A \exp \left\{-a E^{-d / 2}\right\} .
$$

This result was rederived rigorously (including the prefactors and without any assumptions) by many authors [13-19] using different variants of instant on-type calculations in the replicated Hamiltonian or equivalent path-integral methods. The same behaviour was found in classical systems like the attractive RW [19] and the closely related problem of free RW with traps [20, 21]. This latter model on percolating clusters was recently analysed [22, 23]. Both random hopping and random trapping with a singular distribution of the parameters were investigated for $d>1$ [24].

We argue here that for classical diffusion on random clusters the asymptotic behaviour is significantly different from the above mentioned models. Basically, we find that the spectral dimension should replace the spatial one in equation (4) above. The long-time behaviour is dominated by the most ramified clusters and the one-dimensional result (3) will hold in arbitrary dimension (clearly there is only one kind of clusters for $d=1$ ).

To arrive at this conclusion let us look first at the asymptotic behaviour for small frequencies of the density of modes $\rho(\omega)$, the Laplace transform of $\Delta P_{0}(t)$ :

$$
\rho(\omega)=\int_{0}^{\infty} \mathrm{d} t \Delta P_{0}(t) \exp \{-\omega t\} .
$$

From equation (1) we have the following relation between the frequency associated with a given linear length $L$ :

$$
\omega \sim L^{-(2+\theta)}
$$

We then apply our generalized Lifshitz argument [12] to a given cluster specified by its fractal $\left(\bar{d}_{\alpha}\right)$ and spectral $\left(\tilde{d}_{\alpha}\right)$ dimensions. The number of sites (or bonds), $s$, is related to its physical linear length $L$ through the fractal dimension :

$$
s \sim L^{\overline{\mathrm{d}}_{\alpha}} .
$$


We are interested in the $\omega \rightarrow 0$ regime, below the threshold, where the density of states is primarily determined by the probability to find a very large cluster. If the probability for a site (or bond) to be occupied is $p \ll p_{\mathrm{c}}\left(p_{\mathrm{c}}\right.$ is the concentration at the threshold), the probability to form a very large cluster with $s$ sites (or bonds) is given by [4] :

$$
\operatorname{Pr}(s ; \alpha)=A_{\alpha}(s) \exp \left\{-a_{\alpha}(p) s\right\} .
$$

$a_{\alpha}(p)$ is a constant which depends on the intrinsic topology of the clusters in the class $\alpha$, on the spatial dimension, the lattice structure, etc., but primarily on the concentration $p$. The leading behaviour of $A_{\alpha}(s)$ is a power of $s$ which also varies with the class $\alpha$ and the dimensionality.

The central point for our argument is the exponential decay of this probability (9) with $s$. It follows, with the use of (2), (7), (8) and (9), the asymptotic form of the density of modes :

$$
\rho(\omega ; \alpha) \sim B_{\alpha}(\omega) \exp \left\{-b_{\alpha}(p) \omega^{-\tilde{d}_{\alpha / 2}}\right\} .
$$

The same behaviour holds for all clusters within the same class $\alpha$. The ensemble of random clusters consists of many classes with different $\tilde{d}_{\alpha}$ which vary from $\tilde{d}=1$ for the chain-like clusters to $\tilde{d}=d$ for the compact ones. In between lies the largest class of the most frequent clusters which are animal-like and which have a spectral dimension $1<\tilde{d}_{\mathrm{a}}<d$. Whether $\tilde{d}_{\mathrm{a}}=4 / 3$ or some other close value is irrelevant to our argument. The fact that the animals are the most numerous is reflected through their factors $a_{\mathrm{a}}(p)$ in (9) (or $b_{\mathrm{a}}(p)$ in (10)) which are the smaller of all $a_{\alpha}(p)$ $\left(b_{\alpha}(p)\right)$ for a given lattice and concentration $p$. For small enough frequencies, however, it is the spectral dimension $\tilde{d}_{\alpha}$, through the factor $\omega^{-\tilde{d}_{\alpha / 2}}$ in the exponential of equation (10), that will determine which class of clusters will dominate the asymptotic behaviour. Obviously this will be the class with the minimal $\tilde{d}_{\alpha}$, namely the chain-like clusters with $\tilde{d}_{\mathrm{c}}=1$. In other words, we may always find $\omega$ small enough such that $b_{c} \omega^{-1 / 2}$ will be smaller than all others $b_{\alpha} \omega^{-\tilde{d}_{\alpha} / 2}$, namely the difference in the spectral dimensions more than compensate that of the constants $b$ arising from the statistics.

To find the behaviour of $\Delta P_{0}(t)$ it remains to transform back (6) and a simple saddle point calculation yields :

with

$$
\Delta P_{0}(t ; \alpha) \sim C_{\alpha}(t) \exp \left\{-c_{\alpha}(p) t^{\psi_{\alpha}}\right\}
$$

$$
\psi_{\alpha}=\tilde{d}_{\alpha} /\left(2+\tilde{d}_{\alpha}\right) .
$$

If indeed for lattice animals $\tilde{d_{a}} \approx 4 / 3$ then their contribution to $\Delta P_{0}(t)$ with $\psi_{\mathrm{a}} \approx 2 / 5$ will dominate the intermediate time scales. As $t \rightarrow \infty$, however, it is again the term with the minimal $\psi_{\alpha}$ $\left(\psi_{\mathrm{c}}=1 / 3\right.$ of the chains) which will set the leading behaviour as quoted above in equation (3).

One should bare in mind the very asymptotic nature of this result which deals with the tail of $\rho(\omega)$ (or $\left.\Delta P_{0}(t)\right)$. Yet, it is useful to note that the number of $s$-chains increases exponentially with $s$ (the appropriate growth parameter [4] affects the constant $a_{\mathrm{c}}$ in $(9)$, their probability decays exponentially due to the small value of $p$ ). Therefore, the $s$-chains are more frequent than the compact clusters with $s$ sites whose number increases exponentially only with $s^{1-1 / d}$ [4]. The latter, nevertheless, dominate the tail in the quantum case (see above) and provide an example where very untypical configurations make the leading contribution.

The crossover frequency $\omega^{*}$, below which the asymptotic behaviour $\rho(\omega) \sim \exp \left\{-b_{\mathrm{c}} \omega^{-1 / 2}\right\}$ will dominate, is not a universal one in any sense. We may estimate it, for each specific case, by comparing the contribution of the chains to that of the most frequent animal clusters. To get an order of magnitude estimate for the triangular lattice, we may assume $\widetilde{d}_{\mathrm{a}}=4 / 3$ and use other information from the literature [ 4 and references, 25]. For $p=0.05,0.1$ and 0.2 , the respective values of $\omega^{*}(p)$ are 1/3,1/10 and 1/400 (in units of $2 \pi v_{0}$ where $v_{0}$ is the basic hopping frequency). The corresponding $t^{*}$ (in units of the basic time scale $\tau_{0}=1 / v_{0}$ ) for the asymptotic 
behaviour of $\Delta P_{0}(t)^{\circ}$ are 10,40 and 7600 respectively. There is, however, a severe constraint on the size of the cluster necessary to observe this behaviour. For the best case $(p=0.05)$ we may estimate the probability for a cluster of the minimal size to be of the order of $1: 10^{13} ! \rho(\omega)$ and $\Delta P_{0}(t)$ are of the same order of magnitude when the asymptotic behaviour is reached. Presumably it is unobservable in any feasible numerical simulation.

We conclude with a comparison with other models mentioned above. In the system analysed here the minimal, non-vanishing, frequency was set by the largest scale of the cluster under consideration. In the quantum case it is the shortest one which gives (for a given cluster assumed to be regular), due to the uncertainty principle, the lower bound for the eigenenergies. In the same way, it is again the shortest size of the region free from traps which fixes the maximal survival time for random-trapping RW. The most compact (spherical) clusters are indeed the most important ones, for both models, in contrast with the situation of the lost ant discussed here.

\section{Acknowledgments.}

I am thankful to S. Alexander for a valuable correspondence. Relevant references were brought to my attention during useful conversations with A. Aharony, S. Alexander, Y. Gefen, Y. Oono, R. Rammal, G. Toulouse and I. Webman. These discussions took place during visits at Ecole Normale Supérieure (Paris) and at the Institute for Theoretical Physics (UCSB) for which the author is most grateful. This work was supported by the NSF under the MRL grant DMR-8020250 at the University of Illinois and at Brookhaven National Laboratory by The Division of Materials Sciences, U.S. Department of Energy contract DE-ACO2-76CH00016.

Note added. -- After this paper was submitted, I received the preprint of Ref. [25] (for which I am grateful to D. Stauffer). These authors conjecture $\psi=\psi_{\mathrm{a}}=2 / 5$ from scaling arguments and claim to find numerical confirmation for it. They do not consider, however, the special role of non-typical clusters in controlling the asymptotic long-time limit. In spite of the limitations quoted above, it will be interesting to reanalyse their data and to look for a potential trend of $\psi$ becoming smaller on a longer time. This will be an indication that their numerical results only reflect a transient behaviour on the intermediate time scales dominated by the animals. Finally, recent extensive numerical simulations for lattice animals (Havlin, S. et al., Phys. Rev. Lett. 53 (1984) 178) suggest $\tilde{d}_{\mathrm{a}}=1.20$ (in $d=3$ ) which implies $\psi_{\mathrm{a}}=3.75$ and therefore the effective $\psi$ must be smaller than this value in order to reach out from the potential animal-dominated regime.

\section{References}

[1] De Gennes, P. G., La Recherche 7 (1976) 919.

[2] Shender, E. F., J. Phys. C 9 (1976) L-309;

-Mitescu, C., Ottavi, H. and RoussenQ, I., A.I.P. Conf. Proc. 40 (1978) 377.

[3] Straley, J. P., J. Phys. C 13 (1980) 2991.

[4] Stauffer, D., Phys. Rep. 53 (1979) 1.

[5] Gefen, Y., Aharony, A. and AleXander, S., Phys. Rev. Lett. 50 (1983) 77.

[6] Ben-Avraham, D. and Havlin, S., J. Phys. A 16 (1982) L-483.

[7] Pandey, R. B. and Stauffer, D., Phys. Rev. Lett. 51 (1983) 527.

[8] Alexander, S. and Orbach, R., J. Physique Lett. 43 (1982) L-625.

[9] Rammal, R. and Toulouse, G., J. Physique Lett. 44 (1983) L-13.

[10] Alexander, S., Bernasconi, J. and Orbach, R., Phys. Rev. B 17 (1978) 4311.

[11] Tanaka, F., J. Phys. C 13 (1980) L-1.

[12] LifshitZ, I. M., Usp. Fiz. Nauk. 83 (1964) 617 (Sov. Phys. Usp. 7 (1965) 549). 
[13] Halperin, B. I. and LaX, M., Phys. Rev. 148 (1966) 722.

[14] Edwards, S. F., J. Non-Cryst. Solids 4 (1970) 417.

[15] Friedberg, R. and Luttinger, J. M., Phys. Rev. B 12 (1975) 4460.

[16] Cardy, J., J. Phys. C 11 (1978) L-321.

[17] Brezin, E. and Parisi, G., J. Phys. C 13 (1980) L-307.

[18] Sadovskil, M. V., Zh. Eksp. Teor. Fiz. 83 (1982) 1418 (Sov. Phys. JETP 56 (1982) 816).

[19] Donsker, N. D. and Varadhan, S. R. S., Commun. Pure Appl. Math. 28 (1975) 1, 279 and 525.

[20] Grassberger, P. and Procaccia, I., J. Chem. Phys. 77 (1982) 6281.

[21] Kayser, R. F. and Hubbard, J. B., Phys. Rev. Lett. 51 (1983) 79.

[22] De Gennes, P. G., C.R. Hebd. Séan. Acad. Sci., Sér. II 296 (1983) 881.

[23] Webman, I., Phys. Rev. Lett. 52 (1984) 220.

[24] Alexander, S., Phys. Rev. B 23 (1981) 2951.

[25] Pandey, R. B., Stauffer, D., Margolina, A. and Zabolitzky, J. G., J. Stat. Phys. 34 (1984) 427. 